\title{
Hsing-chien Tsai
}

\section{FINITELY INSEPARABLE FIRST-ORDER AXIOMATIZED MEREOTOPOLOGICAL THEORIES}

\begin{abstract}
This paper will first introduce first-order mereotopological axioms and axiomatized theories which can be found in some recent literature and it will also give a survey of decidability, undecidability as well as other relevant notions. Then the main result to be given in this paper will be the finite inseparability of any mereotopological theory up to atomic general mereotopology (AGEMT) or strong atomic general mereotopology (SAGEMT). Besides, a more comprehensive summary will also be given via making observations about other properties stronger than undecidability.
\end{abstract}

Keywords: mereology, mereotopology, decidability, undecidability, separability, inseparability, finite separability, finite inseparability

\section{Mereotopological Axioms and Theories}

The first-order language of mereotopological theories contains only two binary predicates ' $\mathrm{P}$ ' and ' $\mathrm{C}$ ', which stand respectively for "being a part of" and "contact". It is known that the so-called mereological theories are based on ' $\mathrm{P}$ ' only (traditionally, mereology is the theory of the parthood relation). Moreover, ' $\mathrm{C}$ ' is usually viewed as a topological predicate. Thus mereotopological theories can be viewed as extensions of

1 Note that the first-order language considered here also contains the equality sign. The relation "contact" is called "be connected to" by [1]. Such a nomenclature originates from [17]. However, as Pratt-Hartmann has pointed out [6, p. 22], one might confuse such a term with the standard topological notion of "connectedness". To avoid the confusion, the name "contact" is adopted here. 
mereological theories by adding axioms about an additional topological predicate ' $\mathrm{C}$ '. In this light, in this paper the collection of possible mereological axioms and mereological theories will be introduced first and then the way of getting their mereotopological extensions will be shown next. ${ }^{2}$ To make expressions more succinct, four additional predicates are defined as follows.

$$
\begin{aligned}
& \text { (Proper Part:) } \mathrm{PP} x y \stackrel{\mathrm{df}}{=} \mathrm{P} x y \wedge \neg \mathrm{P} y x \\
& \quad \text { (Overlap): } \mathrm{O} x y \stackrel{\text { df }}{=} \exists z(\mathrm{P} z x \wedge \mathrm{P} z y) \\
& \text { (Underlap): } \mathrm{U} x y \stackrel{\mathrm{df}}{=} \exists z(\mathrm{P} x z \wedge \mathrm{P} y z) \\
& \text { (Internal Part): } \mathrm{IP} x y \stackrel{\text { df }}{=} \mathrm{P} x y \wedge \forall z(\mathrm{C} z x \rightarrow \mathrm{O} z y)
\end{aligned}
$$

In the literature $[8,1]$, the following mereological axioms can be found.

$$
\begin{aligned}
& \text { (P1) } \mathrm{P} x x \\
& \text { (P2) }(\mathrm{P} x y \wedge \mathrm{P} y x) \rightarrow x=y \\
& \text { (P3) }(\mathrm{P} x y \wedge \mathrm{P} y z) \rightarrow \mathrm{P} x z \\
& \text { (reflexivity for ' } \mathrm{P} \text { ') } \\
& \text { (anti-symmetry for ' } \mathrm{P} \text { ') } \\
& \text { (transitivity for ' } \mathrm{P} \text { ') } \\
& \text { (EP) } \forall x \forall y(\exists z \mathrm{PP} z x \rightarrow(\forall z(\mathrm{PP} z x \leftrightarrow \mathrm{PP} z y) \rightarrow x=y)) \text { (extensionality) } \\
& \text { (WSP) } \forall x \forall y(\mathrm{PP} x y \rightarrow \exists z(\mathrm{PP} z y \wedge \neg \mathrm{O} z x)) \quad \text { (weak supplementation) } \\
& \text { (SSP) } \forall x \forall y(\neg \mathrm{P} y x \rightarrow \exists z(\mathrm{P} z y \wedge \neg \mathrm{O} z x)) \quad \text { (strong supplementation) } \\
& \text { (FS) } \forall x \forall y(\mathrm{U} x y \rightarrow \exists z \forall w(\mathrm{O} w z \leftrightarrow(\mathrm{O} w x \vee \mathrm{O} w y))) \\
& \text { (FP) } \forall x \forall y(\mathrm{O} x y \rightarrow \exists z \forall w(\mathrm{P} w z \leftrightarrow(\mathrm{P} w x \wedge \mathrm{P} w y))) \\
& \text { (A) } \forall x \exists y(\mathrm{P} y x \wedge \forall z \neg \mathrm{PP} z y) \\
& \text { (A) } \forall x \exists y \operatorname{PP} y x \\
& \text { (G) } \exists x \forall y \text { P } y x \\
& \text { (C) } \forall x(\neg \forall z \mathrm{P} z x \rightarrow \exists z \forall w(\mathrm{P} w z \leftrightarrow \neg \mathrm{O} w x)) \\
& \text { (finite sum) } \\
& \text { (finite product) } \\
& \text { (atomicity) } \\
& \text { (atomlessness) } \\
& \text { (UF) } \exists x \alpha(x) \rightarrow \exists z \forall y(\mathrm{O} y z \leftrightarrow \exists x(\alpha(x) \wedge \mathrm{O} y x)) \\
& \text { (the greatest member) } \\
& \text { (complementation) }
\end{aligned}
$$

A mereological theory can be formed by using some of the axioms listed above and the following are theories which have been named in the literature (the nomenclature here mainly follows [1]). Note that in the literature, the first three axioms (P1), (P2) and (P3) are the most basic (it is said that they constitute part of the meaning of "part"; see [8]) and hence must be included in any mereological theory, which means that any mereological structure must be a partial ordering:

\footnotetext{
2 The way to be considered mainly follows [1].
} 
Ground Mereology: GM $\stackrel{\text { df }}{=}(\mathrm{P} 1)+(\mathrm{P} 2)+(\mathrm{P} 3)$

Minimal Mereology: $\mathbf{M M} \stackrel{\mathrm{df}}{=} \mathbf{G M}+(\mathrm{WSP})$

Extensional Mereology: $\mathbf{E M} \stackrel{\mathrm{df}}{=} \mathbf{G M}+(\mathrm{SSP})$

Closure Mereology: $\mathbf{C M} \stackrel{\mathrm{df}}{=} \mathbf{G M}+(\mathrm{FS})+(\mathrm{FP})$

Minimal Extensional Mereology: $\mathbf{M E M} \stackrel{\mathrm{df}}{=} \mathrm{MM}+(\mathrm{FP})$

Minimal Closure Mereology: $\mathbf{C M M} \stackrel{\mathrm{df}}{=} \mathbf{M M}+(\mathrm{FS})+(\mathrm{FP})$

Extensional Closure Mereology: $\mathbf{C E M} \stackrel{\mathrm{df}}{=} \mathbf{E M}+(\mathrm{FS})+(\mathrm{FP})$

General Extensional Mereology: GEM $\stackrel{\mathrm{df}}{=} \mathbf{E M}+(\mathrm{UF})$

It is easy to show that (EP), (WSP), (FS), (FP), (G) and (C) are theorems of GEM and therefore GEM is the strongest theory on this list. ${ }^{3}$ Actually, GEM $+(\mathrm{A})$ and $\mathbf{G E M}+(\overline{\mathrm{A}})$ are the two strongest incompatible mereological theories which can be formed by using the mereological axioms listed above. Both GEM $+(\mathrm{A})$ and GEM $+(\overline{\mathrm{A}})$ are consistent, for it is easy to check that the former is satisfied by any atomic Boolean algebra with the least member removed and the latter, by any atomless Boolean algebra with the least member removed (if P $x y$ is interpreted by $x \leq y$ in a Boolean algebra).

Before introducing mereotopological axioms, again for the sake of convenience, let's define the following additional function symbols.

(Addition):

$$
x+y=z \text { iff }(\mathrm{U} x y \wedge \forall w(\mathrm{O} w z \leftrightarrow(\mathrm{O} w x \vee \mathrm{O} w y))) \vee(\neg \mathrm{U} x y \wedge x=z)
$$

(Product):

$$
x \times y=z \text { iff }(\mathrm{O} x y \wedge \forall w(\mathrm{P} w z \leftrightarrow(\mathrm{P} w x \wedge \mathrm{P} w y))) \vee(\neg \mathrm{O} x y \wedge x=z)
$$

(Complement):

$$
\sim_{x}=z \operatorname{iff}(\neg \forall z \mathrm{P} z x \wedge \forall w(\mathrm{P} w z \leftrightarrow \neg \mathrm{O} w x)) \vee(\forall z \mathrm{P} z x \wedge x=z)
$$

(Fusion): for any formula $\alpha$ in which ' $x$ ' is free but ' $z$ ' and ' $y$ ' do not occur free

$$
\begin{aligned}
\mathcal{F} x(\alpha(x))=z \text { iff }(\exists x \alpha(x) \wedge \forall y(\mathrm{O} y z \leftrightarrow & \exists x(\alpha(x) \wedge \mathrm{O} y x))) \\
\vee & (\neg \exists x \alpha(x) \wedge \forall y \mathrm{P} y z)
\end{aligned}
$$

${ }^{3}$ GEM is also known in the literature as "classical mereology", which is a decidable theory. By the way, for the results of decidability concerning those mereological theories, please see $[12,13,14,15]$. 
(Interior):

$$
\mathrm{i}(x)=z \operatorname{iff}(\exists y \operatorname{IP} y x \wedge \mathcal{F} y(\operatorname{IP} y x)=z) \vee(\neg \exists y \operatorname{IP} y x \wedge \forall y \operatorname{P} y z)
$$

(Exterior):

$$
\mathrm{e}(x) \stackrel{\mathrm{df}}{=} \mathrm{i}(\sim x)
$$

(Closure):

$$
\mathrm{c}(x) \stackrel{\mathrm{df}}{=} \sim(\mathrm{e}(x))
$$

(Boundary):

$$
\mathrm{b}(x) \stackrel{\mathrm{df}}{=} \sim(\mathrm{e}(x)+\mathrm{i}(x))
$$

Note. These definitions basically follow [1]. However, their original definitions define only partial functions. Here the definitions are modified in a harmless way so as to make each function total if the theory considered is sufficiently strong (more precisely, if the theory considered is not weaker than EM, $\forall z(O x z \rightarrow O y z) \rightarrow P x y$ will be a theorem; then by (P2) and such a theorem, it is easy to see the uniqueness of the $z$ in each definition above). Note that the fusion function symbol ' $\mathcal{F} x$ ' is actually a meta-function symbol which takes formulas as parameters. Therefore, formally we shall take $\mathcal{F} x(\alpha(x))=z$ as an abbreviation for its definition.

Now the list of possible mereotopological axioms will include the mereological axioms mentioned earlier and the following ones.
(C1) $\mathrm{C} x x$
(C2) $\mathrm{C} x y \rightarrow \mathrm{C} y x$
(C3) $\mathrm{P} x y \rightarrow \forall z(\mathrm{C} z x \rightarrow \mathrm{C} z y)$
(C4) $\forall z(\mathrm{C} z x \rightarrow \mathrm{C} z y) \rightarrow \mathrm{P} x y$
(B) $\forall x \exists y(\operatorname{IP} y x \wedge \neg \operatorname{IP} x y)$
(K1) $\mathrm{P} x \mathrm{c}(x)$
$(\mathrm{K} 2) \mathrm{c}(\mathrm{c}(x))=\mathrm{c}(x)$
(K3) $\mathrm{c}(x+y)=\mathrm{c}(x)+\mathrm{c}(y)$

(reflexivity for ' $\mathrm{C}$ ') (symmetry for ' $\mathrm{C}$ ')

(monotonicity)

(defining ' $\mathrm{P}$ ')

(boundarylessness)

Note that the first three axioms (C1), (C2) and (C3) are the most basic and must be included in any mereotopological theory and that (K1), (K2) and (K3) are actually mereotopological counterparts of Kuratowski's axioms for topological closures. The following nomenclature of mereotopological theories is given by [1]. Suppose $\mathbf{X}$ is a mereological theory. $\mathbf{X}+(\mathrm{C} 1)+(\mathrm{C} 2)+(\mathrm{C} 3)$ will be named $\mathbf{X T}$ (if $\mathbf{X}$ is $\alpha$-mereology, $\mathbf{X T}$ will be read as $\alpha$-mereotopology; for instance, EMT is extensional mereotopology). Furthermore, for any $\mathbf{X T}, \mathbf{X T}+(\mathbf{A})$ is named AXT, $\mathbf{X T}+(\overline{\mathrm{B}})$ is named $\overline{\mathbf{B}} \mathbf{X T}$ and $\mathbf{X T}+(\overline{\mathrm{A}})$ is named $\overline{\mathbf{A}} \mathbf{X} \mathbf{T}$. Besides, GEMT $+(\mathrm{K} 1)+(\mathrm{K} 2)+(\mathrm{K} 3)$ is named GEMTC, GEMTC $+(\mathrm{A})$ is 


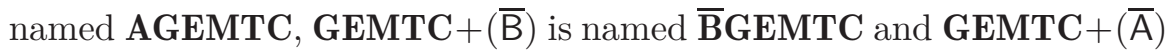
is named $\mathbf{A}$ GEMTC. ${ }^{4}$ In this setting, there are two incompatible maxi-

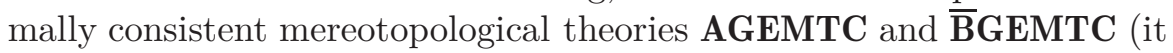
is easy to see that $\overline{\mathbf{B}}$ GEMTC implies $\overline{\mathrm{A}}$ and that no finite model can

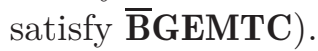

So far (C4) has not been included in any mereotopological theory defined above. If ( $\mathrm{C} 4)$ is adopted, then together with (C3), we will have a definition of ' $\mathrm{P}$ ' in terms of ' $\mathrm{C}$ ', that is to say, the formal language will then in effect have only one primitive, viz. 'C'. Now, for any mereotopological theory $\mathbf{X}$ which does not have (C4), $\mathbf{X}+(\mathrm{C} 4)$ will be named $\mathbf{S X}$ (Strong $\mathbf{X}$ ).

There is a variation due to [2], which defines fusion by "contact" instead of "overlap". Such a variation replaces principles (FS), (FP), (C) and (UF) by the following versions (then all the function symbols defined above will also have to be redefined accordingly).

$$
\begin{gathered}
\left(\mathrm{FS}^{\prime}\right) \forall x \forall y(\mathrm{U} x y \rightarrow \exists z \forall w(\mathrm{C} w z \leftrightarrow \exists u((\mathrm{P} u x \vee \mathrm{P} u y) \wedge \mathrm{C} w u))) \\
\left(\mathrm{FP}^{\prime}\right) \forall x \forall y(\mathrm{O} x y \rightarrow \exists z \forall w(\mathrm{C} w z \leftrightarrow \exists u(\mathrm{P} u x \wedge \mathrm{P} u y \wedge \mathrm{C} w u))) \\
\quad \text { (finite product for 'C') } \\
\left(\mathrm{C}^{\prime}\right) \forall x(\neg \forall z \mathrm{P} z x \rightarrow \exists z \forall w(\mathrm{P} w z \leftrightarrow \neg \mathrm{C} w x)) \quad \text { (complementation for 'C') } \\
\left(\mathrm{UF}^{\prime}\right) \exists x \alpha(x) \rightarrow \exists z \forall y(\mathrm{C} y z \leftrightarrow \exists \\
\text { (unrestricted fusion axiom schema for 'C') }
\end{gathered}
$$

For any formula $\alpha$ in which ' $x$ ' is free but ' $z$ ' and ' $y$ ' do not occur free. Note that $\alpha$ might have free variables other than ' $x$ '.

Then for any theory SX, if (FS), (FP), (C) and (UF) in SX are replaced respectively by $\left(\mathrm{FS}^{\prime}\right),\left(\mathrm{FP}^{\prime}\right),\left(\mathrm{C}^{\prime}\right)$ and $\left(\mathrm{UF}^{\prime}\right)$, the resultant theory will be named $\mathbf{S X}^{\prime}$, for example, replacing (UF) in GEMT by $\left(\mathbf{U F}^{\prime}\right)$ will yield GEMT'$^{\prime}$. As mentioned earlier, AGEMTC and BGEMTC are the two maximally consistent theories if (C4) has not been adopted, so it would be a good and actually correct guess that the two maximally consistent theories which can be formed by adding (C4) as an axiom are SAGEMTC

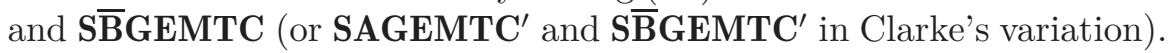

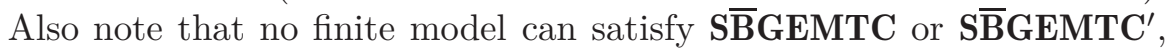
which is a fact not difficult to see. than GEM.

${ }^{4}[1]$ did not consider $\mathbf{X T}+(\mathrm{K} 1)+(\mathrm{K} 2)+(\mathrm{K} 3)$ for any $\mathbf{X}$ which is strictly weaker 
The foregoing sets up the scope of the mereotopological axioms and theories which will be considered in this paper.

\section{Decidability and Other Relevant Notions}

First of all, note that here we are only concerned with consistent theories. Traditionally, decidability is an important issue for metalogical researches. A logical theory $T$ is decidable if and only if there is an effective procedure via which one can check whether a sentence is a theorem of $T$ or not. Of course, "effective procedure" itself still calls for elucidation. It has been characterized in the literature by the following three conditions [3, p. 61]: first, the execution of an effective procedure can be explained by exact instructions; second, the instructions of an effective procedure can be mechanically implemented without any random devices; third, for any input, an effective procedure will output an answer, no matter whether it is positive or negative, within finitely many steps. It seems that in order to prove the decidability of a logical theory, one has to come up with an effective procedure for checking the theorems of that theory. However, in many cases, it is really hard to find a substantial theorem-checking procedure for the theory considered. Instead, it is quite often that a proof will be carried out by a "reduction", that is, taking a theory/theories which is/are known decidable (call it/them "basis theory/theories") and trying to relate in some way the theory under investigation (call it "target theory") to the basis theory/theories.

Reduction is also an important (perhaps the most important) way for proving the undecidability of a theory (it goes without saying that a theory is undecidable if and only if it is not decidable). As for in what way the target theory should be related to the basis theory/theories, it depends on which general metalogical theorem for proving undecidability we are applying in that case. Some useful general metalogical theorems for proving undecidability will be introduced below.

Furthermore, the reductions involved are quite often model theoretical, that is, in those cases, the way to relate the target theory to the basis theory/theories is the way to relate the models of the former to the models of the latter. Normally, a model theoretical reduction relies on a general way of interpreting or defining a model of a theory into a model of another. Some examples will be given in the next section. 
Moreover, in almost all cases, the syntactical items, such as symbols, terms, well-formed formulas and finite sequences of well-formed formulas, of the formal language considered can be coded into natural numbers in an effective way. That is to say, we can define a one-to-one function from the sequences of symbols of the formal language considered to natural numbers such that given a sequence of symbols, we can effectively determine the natural number which such a sequence is mapped to and, given a natural number, we can effectively determine whether it is a code of a sequence of symbols or not and if the answer is positive, we can further effectively determine which sequence is coded by that number. In this light, the question whether a logical theory $T$ is decidable or not amounts to the question whether the set of natural numbers each of which is the code of a theorem of $T$ is decidable or not (henceforth, to follow a prevailing logical jargon, a code of the said kind will be called a Gödel number). However, owing to Church's thesis, a set of natural numbers is decidable if and only if its characteristic function is recursive. Therefore, as we will see, some general metalogical theorems for proving undecidability have resorted to notions and theorems of the recursion theory. ${ }^{5}$

Intuitively, decidable theories are easier for us to handle (here we do not consider the issue of complexity) while undecidable theories are tougher. Based on how they "misbehave", undecidable theories can be further classified into a few subclasses. The following are definitions of those subclasses which can be found in the literature.

- A theory $T$ is essentially undecidable if and only if all its consistent extensions in the same language are undecdiable.

- A theory $T$ is hereditarily undecidable if and only if all its subtheories are undecidable.

- A theory $T$ is strongly undecidable in the first sense if and only if any theory in the same language which is compatible with $T$ is undecidable.

Before introducing other subclasses of undecidable theories, let's see some notions that we need from the recursion theory.

- Two sets $A$ and $B$ of natural numbers are recursively inseparable if and only if there is no recursive set $C$ such that $A \subseteq C$ and $B \subseteq \omega \backslash C$.

${ }^{5}$ For a nice expository remark on Church's thesis, please see [3, pp. 206-210]. A recursive function is a total partial recursive function and the definition of "partial recursive function" can be found in any prestigious textbook of the recursion theory. For a quick but clear introduction, please see [9, Ch. 1]. 
- Two sets $A$ and $B$ of natural numbers are effectively inseparable if and only if they are disjoint and there is a binary recursive function $f$ such that, for any two disjoint recursively enumerable sets $C$ and $D$ such that $A \subseteq C, B \subseteq D, f(c, d) \notin C \cup D$, where $c$ and $d$ are indices of $C$ and $D$ respectively. ${ }^{6}$

Then other subclasses of undecidable theories are as follows.

- A theory $T$ in a language $L$ is strongly undecidable in the second sense if and only if $\{\# \alpha: \alpha \in L$ and $T \vdash \alpha\}$ and $\{\# \alpha: \alpha \in$ $L$ and $T \cup\{\neg \alpha\}$ has a finite model $\}$ are recursively inseparable, where $\alpha$ is a formula and $\# \alpha$ is the Gödel number of $\alpha$ (note that here $\alpha$ does not have to be a sentence).

- A theory $T$ in a language $L$ is inseparable if and only if the set of Gödel numbers of the theorems of $T$ and the set of Gödel numbers of the sentences whose negations are theorems of $T$ are effectively inseparable.

- A theory $T$ in a language $L$ is finitely inseparable if and only if the set of Gödel numbers of the valid sentences in $L$ and the set of Gödel numbers of the sentences each of which can be refuted by some finite model of $T$ are effectively inseparable. ${ }^{7}$

${ }^{6}$ A set of natural numbers is recursively enumerable (abbreviated as r.e.) if and only if it is the domain of some partial recursive function. Intuitively, a set is r.e. if and only if there is an effective procedure via which members of such a set can be enumerated one by one, that is, via such a procedure, we can "count" the set in question: the first member is [...], the second member is [...], and so on. In this light, it is easy to see that if a set of natural numbers is decidable, then it must be r.e. As for indices of an r.e. set, by Church's thesis, partial recursive functions are exactly Turing computable functions, where a Turing computable function is a function which can be computed by a Turing program, and it is obvious that the same partial recursive function can be computed by infinitely many Turing programs (just add to a program some redundant instructions and then it will still do the same job), but Turing programs can be mapped one-to-one and onto natural numbers, so each partial recursive function will have infinitely many natural numbers as its indices. Here the indices of an r.e. set are exactly the indices of the partial recursive function whose domain is the r.e. set in question.

7 The definitions of "essentially undecidable" and "hereditarily undecidable" are given by [10]. The definition of "strongly undecidable" given by [3] is what I mean by the "strongly undecidable in the first sense" and the definition of "strongly undecidable" given by [4] is what I mean by the "strongly undecidable in the second sense". The definitions of "essentially undecidable" and "strongly undecidable" are formulated by [7] in terms of models, but they are equivalent respectively to "essentially 
To see that a theory $T$ of any of the foregoing three kinds is undecidable, observe the following three facts. First, if a theory $T$ is decidable, $\{\# \alpha: \alpha \in L$ and $T \vdash \alpha\}$ will also be decidable and hence r.e. (observe that $T \vdash \alpha$ if and only if the universal closure of $\alpha$, which is a sentence, is a theorem of $T$ ), and so will be its complement (if a set of natural numbers is decidable, it is trivial that its complement is also decidable, for we can use the same effective procedure except that the output will be reversed). Second, obviously, the set of Gödel numbers of the valid sentences in $L \subseteq$ the set of Gödel numbers of the theorems of $T \subseteq\{\# \alpha: \alpha \in L$ and $T \vdash \alpha\}$. Third, $\{\# \alpha: \alpha \in L$ and $T \cup\{\neg \alpha\}$ has a finite model $\}$, the set of Gödel numbers of the sentences whose negations are theorems of $T$ and the set of Gödel numbers of the sentences each of which can be refuted by some finite model of $T$ are subsets of the complement of $\{\# \alpha: \alpha \in L$ and $T \vdash \alpha\}$. Then, since the union of any subset of natural numbers and its complement must be the set of all natural numbers, we can see from the said facts and the relevant definitions above that if $T$ is decidable, $T$ can be neither strongly undecidable in the second sense nor inseparable nor finitely inseparable.

Any of the aforementioned subclasses of undecidable theories is defined by a property stronger than undecidability. Such a property seems naturally interesting, for instance, whether an undecidable theory has a decidable proper extension or a decidable proper subtheory is a question which can naturally come to our mind. ${ }^{8}$

Intuitively, when trying to prove that a theory is undecidable, we might first want to consider those properties which make reference to finite models. This is because finite models are in nature much easier to envisage or construct, which could help a lot with carrying out a reduction. In particular, "finitely inseparable" is a property which might be considered first if the target theory has finite models. Now the following

undecidable" and "strongly undecidable in the first sense" defined above. Finally, the definitions of "inseparable" and "finitely inseparable" are given by [5].

8 Each of these properties in a sense reveals how "terribly" undecidable a theory is. For instance, if a theory $T$ is strongly undecidable in the second sense, the theory of all finite models of $T$ is also undecidable [4, p. 235]. If a theory is finitely inseparable, the theory of all finite models of $T$ cannot be any finitely axiomatizable extension of $T$ [5, p. 267]. If a theory is inseparable, it must be essentially undecidable and moreover, there is an effective procedure $P$ such that given any consistent axiomatizable extension $T^{\prime}$ of $T$, we can find via $P$ a sentence $\alpha$ such that $\alpha \notin T^{\prime}$ and $\neg \alpha \notin T^{\prime}$, that is to say, inseparable theories are effectively incomplete [ibid.] All the said facts can be easily seen from the definitions of the notions involved. 
lemma is a powerful general method for proving the finite inseparability of a theory by model theoretical reduction.

Lemma 1. Let $T$ and $T^{\prime}$ be two theories in languages $L$ and $L^{\prime}$ respectively. Assume that $L$ has only finitely many function symbols. Suppose $L$ can be interpreted into a finitely axiomatized $L^{\prime}$-theory $S^{\prime}$. Call this interpretation $I$. If for each finite model $\mathcal{A}$ of $T$ there is a finite model $\mathcal{B}$ of $T^{\prime} \cup S^{\prime}$ such that $\mathcal{A}=\mathcal{B}^{I}\left(\mathcal{B}^{I}\right.$ is the L-structure defined in the $L^{\prime}$-structure $\mathcal{B}$ by using interpretation $I$ ) and $T$ is finitely inseparable, then $T^{\prime}$ is finitely inseparable too. ${ }^{9}$

If all the theories considered are in the same language, it is more convenient to use the following lemma.

Lemma 2. Assume that $T$ and $T^{\prime}$ are theories in the same language. Suppose that every finite model $\mathcal{M}$ of $T$ can be in some way extended to a finite model $\mathcal{M}^{\prime}$ of $T^{\prime}$ and that there is a formula $\alpha(x)$ with only one free variable ' $x$ ' such that the set of elements added (if any) to the domain of $\mathcal{M}$ is definable by $\alpha(x)$ in $\mathcal{M}^{\prime}$. Then if $T$ is finitely inseparable, $T^{\prime}$ is finitely inseparable too. ${ }^{10}$

From Lemma 2, it can be easily seen that if $T$ and $T^{\prime}$ are theories in the same language, $T$ is finitely inseparable and every finite model of $T$ is also a model of $T^{\prime}$, then $T^{\prime}$ is finitely inseparable too.

\section{Finite Inseparability of AGEMT and SAGEMT}

First observe that it follows immediately from the definition of finite inseparability that a theory which has no finite model cannot be finitely inseparable. Therefore, any mereotopological theory whose axioms contain $(\overline{\mathrm{A}})$ or $(\overline{\mathrm{B}})$ is not finitely inseparable. However, as will be shown in the following theorem, it turns out that a lot of mereotopological theories each of which has finite models are finitely inseparable.

Theorem 1. All mereotopological theories up to AGEMT or SAGEMT are finitely inseparable.

9 The original lemma is given by [5, p. 272], but the version presented here has been rephrased by the present writer to make it more readable. For how to interpret a language into a theory, please see [3, Sect. 2.7].

10 This is actually a corollary of Lemma 1 . For its proof, please see [13, pp. 255$256]$. 
Proof. By Lemma 2, it is trivial that if a theory is finitely separable, all its subtheories (no matter whether they are axiomatizable or not) in the same language will also be finitely inseparable. Hence it suffices to show that AGEMT and SAGEMT are finitely inseparable. ${ }^{11}$ As mentioned earlier, it is very often that such a kind of proofs will be done by reductions and here the idea is to make use of Lemma 1.

Furthermore, it is known that for any language $L$ whose signature contains only one binary predicate $R$, the theory of $L$, that is, the set of valid sentences in $L$, is finitely inseparable [5, p. 279]. Consider a language $L$ of the said kind. The reductions to be carried out will take the theory of $L$ as the basis.

(T1.1) Finite Inseparability of AGEMT. Let's first take AGEMT as the target theory. In view of Lemma 1, we shall give an interpretation $I$ of $L$ first and then show that any finite $L$-structure $\mathcal{A}$ will be $\mathcal{B}^{I}$, for some finite model $\mathcal{B}$ of AGEMT. Now the interpretation $I$ is defined as follows.

- $\mathrm{I}(\forall)=x$ is an atom and $x$ contacts at least four and at most five other atoms each of which does not contact any other atom different from $x$.

- $I(R)=(x=y$ and $x$ is an atom and $x$ contacts exactly five other atoms each of which does not contact any other atom different from $x$ ) or ( $x$ is an atom and $x$ contacts at least four other atoms each of which does not contact any other atom different from $x$, and $y$ is an atom and $y$ contacts at least four other atoms each of which does not contact any other atom different from $y$, and there are two atoms which contact each other such that $x$ contacts both of them and $y$ contacts at least one of them)

Let 'At $x$ ' be an abbreviation for ' $\neg \exists y$ PP $y x$ '. Then formally

- $\mathrm{I}(\forall)=\operatorname{At} x \wedge \exists y_{1} \exists y_{2} \exists y_{3} \exists y_{4}\left(\operatorname{At} y_{1} \wedge \operatorname{At} y_{2} \wedge \operatorname{At} y_{3} \wedge\right.$ At $y_{4} \wedge y_{1} \neq y_{2} \wedge$ $y_{1} \neq y_{3} \wedge y_{1} \neq y_{4} \wedge y_{2} \neq y_{3} \wedge y_{2} \neq y_{4} \wedge y_{3} \neq y_{4} \wedge x \neq y_{1} \wedge x \neq y_{2} \wedge$

11 Since AGEMT is a subtheory of SAGEMT, I could have only shown the finite separablility of the latter. However, my original proof has been developed by trying to extend a binary relational structure into a finite model of AGEMT in a graphic way and then trying to modify the graphic interpretation so as to make (C4) satisfied. I think a proof will be more readable if the ideas behind the proof can be seen at least to some extent, even if not completely, from its content. So I have preserved the original proof in which the case of AGEMT has been dealt with first. 


$$
\begin{aligned}
& x \neq y_{3} \wedge x \neq y_{4} \wedge \mathrm{C} x y_{1} \wedge \mathrm{C} x y_{2} \wedge \mathrm{C} x y_{3} \wedge \mathrm{C} x y_{4} \wedge \forall u((\text { At } u \wedge \\
& u \neq x) \rightarrow\left(( u \neq y _ { 1 } \rightarrow \neg \mathrm { C } u y _ { 1 } ) \wedge ( u \neq y _ { 2 } \rightarrow \neg \mathrm { C } u y _ { 2 } ) \wedge \left(u \neq y_{3} \rightarrow\right.\right. \\
& \left.\left.\left.\neg \mathrm{C} u y_{3}\right) \wedge\left(u \neq y_{4} \rightarrow \neg \mathrm{C} u y_{4}\right)\right)\right) \wedge \forall z_{1} \forall z_{2}\left(\left(\operatorname{At} z_{1} \wedge \text { At } z_{2} \wedge z_{1} \neq x \wedge\right.\right. \\
& z_{2} \neq x \wedge z_{1} \neq y_{1} \wedge z_{1} \neq y_{2} \wedge z_{1} \neq y_{3} \wedge z_{1} \neq y_{4} \wedge z_{2} \neq y_{1} \wedge z_{2} \neq y_{2} \wedge \\
& z_{2} \neq y_{3} \wedge z_{2} \neq y_{4} \wedge \mathrm{C} x z_{1} \wedge \mathrm{C} x z_{2} \wedge \forall u((\text { At } u \wedge u \neq x) \rightarrow \\
& \left.\left.\left.\left.\left(\left(u \neq z_{1} \rightarrow \neg \mathrm{C} u z_{1}\right) \wedge\left(u \neq z_{2} \rightarrow \neg \mathrm{C} u z_{2}\right)\right)\right)\right) \rightarrow z_{1}=z_{2}\right)\right)
\end{aligned}
$$

Let $\alpha(x)$ be an abbreviation for the foregoing formula and $\beta(x)$ be an abbreviation for a formula which says that $x$ is an atom and $x$ contacts exactly five other atoms each of which does not contact any other atom different from $x$ (from the formal version of $\mathrm{I}(\forall)$, one should know how to write down such a formula). Then formally

- $\mathrm{I}(\mathrm{R})=(x=y \wedge \beta(x)) \vee(\alpha(x) \wedge \alpha(y) \wedge \exists u \exists z($ At $u \wedge$ At $z \wedge u \neq z \wedge$ $\mathrm{Cuz} \wedge \mathrm{C} x u \wedge \mathrm{C} x z \wedge \mathrm{C} y u))^{12}$

Let $\gamma(x, y)$ be an abbreviation for this formula. To see that the foregoing interpretation really works, we shall first show how to extend each finite $L$-structure to a finite model of AGEMT. Now given a finite $L$-structure $\mathcal{A}$, we will construct in the following way a finite model $\mathcal{B}$ of AGEMT step by step.

(Step 1) Take all members in $\operatorname{Dom}(\mathcal{A})$ as atoms in $\operatorname{Dom}(\mathcal{B})$ and for each $a \in \operatorname{Dom}(\mathcal{A})$, add four new atoms $a_{1}, a_{2}, a_{3}$ and $a_{4}$ to $\operatorname{Dom}(\mathcal{B})$ and let $a$ contact $a_{1}, a_{2}, a_{3}$ and $a_{4}$, that is, let $\left(a, a_{1}\right),\left(a, a_{2}\right),\left(a, a_{3}\right)$ and $\left(a, a_{4}\right) \in C^{\mathcal{B}}$. The construction should be such that if $a, b \in \operatorname{Dom}(\mathcal{A})$ and $a \neq b$, then the atoms newly added for them are all distinct.

(Step 2) For any $a \in \operatorname{Dom}(\mathcal{A})$, if $(a, a) \in R^{\mathcal{A}}$, that is, $R(a, a)$ is true in $\mathcal{A}$, then add one more new atom $a_{5}$ and let $\left(a, a_{5}\right) \in C^{\mathcal{B}}$; otherwise, do nothing.

(Step 3) For any distinct $a, b \in \operatorname{Dom}(\mathcal{A})$, if both $(a, b)$ and $(b, a) \in$ $R^{\mathcal{A}}$, then add two new atoms $c$ and $d$ to $\operatorname{Dom}(\mathcal{B})$ such that $c$ contacts $d$ and that $a$ as well as $b$ contacts both $c$ and $d$, that is, let $(c, d),(a, c)$, $(a, d),(b, c)$ and $(b, d) \in C^{\mathcal{B}}$; if $(a, b) \in R^{\mathcal{A}}$ but $(b, a) \notin R^{\mathcal{A}}$, then add two new atoms $c$ and $d$ such that $c$ contacts $d$ and that $a$ contacts both $c$ and $d$ but $b$ contacts only one of them, that is, let $(c, d),(a, c),(a, d)$ and $(b, c) \in C^{\mathcal{B}}$; otherwise, do nothing. Note that the newly added $c$ and $d$ at this step won't contact any other atom other than the ones specified

12 Here $\alpha(x)$ actually says more than we need, for we only need a formula which says " $x$ contacts at least four other atoms each of which does not contact any other atom different from $x$ ", but this does not matter since $x$ must satisfy $\alpha(x)$ anyway. 
above (in this light, observe that each of $c$ and $d$ contacts at most three other atoms).

(Step 4) Take all atoms got so far and construct a finite Boolean algebra (it should be clear that there can be only finitely many atoms after the execution of Step 3) and let $\operatorname{Dom}(\mathcal{B})$ contain exactly all members except 0 of that finite Boolean algebra. As for the interpretations of ' $\mathrm{P}$ ' and ' $\mathrm{C}$ ' in $\mathcal{B}$, let $P^{\mathcal{B}}=\{(a, b) \in \operatorname{Dom}(\mathcal{B}) \times \operatorname{Dom}(\mathcal{B}): a \leq b$ in the said finite Boolean algebra $\}$ and $C^{\mathcal{B}}=\{(a, b) \in \operatorname{Dom}(\mathcal{B}) \times \operatorname{Dom}(\mathcal{B}): a=b$ or $a$ contacts $b$ from the construction at the previous steps or $(b, a) \in C^{\mathcal{B}}$ or for some $d \in \operatorname{Dom}(\mathcal{B}),(d, a) \in P^{\mathcal{B}}$ and $\left.(b, d) \in C^{\mathcal{B}}\right\} .{ }^{13}$

We can see from the foregoing construction the following three facts. First, if $a, b \in \operatorname{Dom}(\mathcal{A})$ and $a \neq b$, then $(a, b) \notin C^{\mathcal{B}}$. Second, for any $a \in \operatorname{Dom}(\mathcal{A})$, each of the atoms added at Step 1 or Step 2 for $a$ does not contact any atom other than itself or $a$. Third, for any atom $a \in \operatorname{Dom}(\mathcal{B})$, if $a$ does not come from $\operatorname{Dom}(\mathcal{A})$, then $a$ won't contact more than three other atoms.

Now we will check that the mereotopological structure $\mathcal{B}$ constructed through the foregoing four steps is a model of AGEMT. Since $\mathcal{B}$ is a finite Boolean algebra with 0 removed, $\mathcal{B}$ satisfies $(\mathrm{A})$ and GEM. ${ }^{14}$ Furthermore, from the definition of $C^{\mathcal{B}}$, we can see that $(\mathrm{C} 1)$ and $(\mathrm{C} 2)$ are obviously satisfied by $\mathcal{B}$. To see that $\mathcal{B}$ satisfies (C3), observe that by the definition of $C^{\mathcal{B}}$, for any $a, b$ in $\mathcal{B}$, if $a$ is a part of $b$, that is, $(a, b) \in P^{\mathcal{B}}$, then for any $d$ in $\mathcal{B}$ such that $d$ contacts $a$, that is, $(d, a) \in C^{\mathcal{B}},(d, b)$ will be in $C^{\mathcal{B}}$ too, that is, $d$ will also contact $b$. So $\mathcal{B}$ is indeed a model of AGEMT. Therefore, given any $L$-structure $\mathcal{A}$, we can get a model $\mathcal{B}$ of AGEMT from the construction above. But $\mathcal{B}^{I}$ will be exactly $\mathcal{A}$. First of all, $\operatorname{Dom}\left(\mathcal{B}^{I}\right)=\{a \in \operatorname{Dom}(\mathcal{B}): \mathcal{B} \models$ $\alpha(x)[a]$, that is, $\alpha(x)$ is true of $a$ in $\mathcal{B}\}$, but from the construction above, we can see that $B \models \alpha(x)[a]$ if and only if $a \in \operatorname{Dom}(\mathcal{A})$ (more precisely, from the aforementioned third fact, we can see that $a$ is an atom and $a$ contacts at least four other atoms if and only if $a \in \operatorname{Dom}(\mathcal{A}))$ and hence $\operatorname{Dom}\left(\mathcal{B}^{I}\right)=\operatorname{Dom}(\mathcal{A})$. Moreover, the interpretation of $R$ in $\mathcal{B}^{I}$ is $\{(a, b) \in$ $\left.\operatorname{Dom}\left(\mathcal{B}^{I}\right) \times \operatorname{Dom}\left(\mathcal{B}^{I}\right): \mathcal{B} \models \gamma(x, y)[a, b]\right\}$. But by Step 3, obviously $\mathcal{B} \models \gamma(x, y)[a, b]$ if and only if $(a, b) \in R^{\mathcal{A}}$ and hence the interpretation

13 The definition of $C^{\mathcal{B}}$ seems circular, but this won't cause any problem, for it is actually an abbreviation for the formal inductive definition of $C^{\mathcal{B}}$.

14 This is a fact already mentioned by [11] a long time ago. But actually the case of finite Boolean algebras is easy to check. Just note that every finite Boolean algebra must be complete and hence the fusion schema will be satisfied. 
of $R$ in $\mathcal{B}^{I}$ is exactly $R^{\mathcal{A}}$. Therefore, as an $L$ - structure, $\mathcal{B}^{I}$ is exactly $\mathcal{A}$. Finally, consider the theory axiomatized by AGEMT $+\exists x \alpha(x)$. It is immediate that $L$ can be interpreted into the theory axiomatized by $\exists x \alpha(x) .{ }^{15}$ Moreover, it is obvious that the structure $\mathcal{B}$ constructed above satisfies AGEMT $+\exists x \alpha(x)$. Then by Lemma 1, AGEMT is finitely inseparable.

(T1.2) Finite Inseparability of SAGEMT. As for the case of SAGEMT, observe that the structure $\mathcal{B}$ constructed above does not necessarily satisfy (C4), for there might be two objects $a$ and $b$ in $\mathcal{B}$ such that everything which contacts $a$ also contacts $b$ but $a$ is not a part of $b$. More precisely, when a finite model $\mathcal{M}$ of AGEMT has at least two members, $\mathcal{M}$ won't satisfy (C4) if there are two distinct atoms in $\mathcal{M}$ which contact each other. To see this, suppose $\mathcal{M}$ is a model of AGEMT and $\operatorname{Dom}(\mathcal{M})$ has $n$ atoms, for some $n \geq 2$. Let $a \in \operatorname{Dom}(\mathcal{M})$ be the object composed of some $n-1$ atoms (the existence of $a$ is guaranteed by the fusion axiom schema $(\mathrm{F}))$, and let $b \in \operatorname{Dom}(\mathcal{M})$ be the only atom which does not belong to $a$. Obviously, $b$ is not a part of $a$ and therefore $b$ cannot contact any part of $a$ if $\mathcal{M}$ satisfies (C4), for otherwise, every object which $b$ contacts must contact $a$ (any object which has $b$ as a proper part will contain other atoms and hence will overlap $a$, but overlapping implies contacting) and hence by ( $\mathrm{C} 4), b$ will be a part of $a$. The foregoing argument applies to every atom in $\mathcal{M}$, which implies that no two atoms in $\mathcal{M}$ can contact each other if $\mathcal{M}$ satisfies (C4). In order to solve this problem, a similar but more complicated construction will be offered. We will interpret $L$ in almost the same manner as in the case of AGEMT except that instead of using atoms, this time we will use objects each of which is composed of exactly two atoms to stand for members in the original $L$-structure and we will make sure that any two atoms in the structure to be constructed won't contact each other.

Let $\delta(x)$ be an abbreviation for $\exists y \exists z($ At $y \wedge$ At $z \wedge y \neq z \wedge \mathrm{P} y x \wedge \mathrm{P} z x \wedge$ $\forall u(\mathrm{PP} u x \rightarrow(u=y \vee u=z)))$, which says that $x$ is composed of exactly two atoms, and for any formula $\varphi$, let $\exists_{n \leq x} \varphi, \exists_{n \geq x} \varphi$ and $\exists_{n=x} \varphi$, where $n \geq 1$, be abbreviations respectively for "at least $n$ objects such that $\varphi$ ", "at most $n$ objects such that $\varphi$ " and "exactly $n$ objects such that $\varphi$ ". It is known from the first course in logic that the said three kinds of expressions in English can be easily translated into formal formulas in logic. Then let's define the interpretation $I$ as follows.

15 If readers wonder why this is immediate, please see [3, Sect. 2.7] again. 
- $\mathrm{I}(\forall)=\delta(x) \wedge \exists_{4 \leq y}(\delta(y) \wedge \mathrm{C} y x \wedge \neg \mathrm{O} y x \wedge \forall z((\delta(z) \wedge z \neq x \wedge \neg \mathrm{O} z y) \rightarrow$ $\neg \mathrm{C} y z)) \wedge \exists_{5 \geq y}(\delta(y) \wedge \mathrm{C} y x \wedge \neg \mathrm{O} y x \wedge \forall z((\delta(z) \wedge z \neq x \wedge \neg \mathrm{O} z y) \rightarrow \neg \mathrm{C} y z))$ - $\mathrm{I}(\mathrm{R})=\left(x=y \wedge \exists_{5=z}(\delta(z) \wedge \mathrm{C} z x \wedge \neg \mathrm{O} z x \wedge \forall u((\delta(u) \wedge u \neq x \wedge \neg \mathrm{O} u z) \rightarrow\right.$ $\neg \mathrm{C} u z))) \vee\left(\exists_{4 \leq z}(\delta(z) \wedge \mathrm{C} z x \wedge \neg \mathrm{O} z x \wedge \forall u((\delta(u) \wedge u \neq x \wedge \neg \mathrm{O} u z) \rightarrow\right.$ $\neg \mathrm{C} u z)) \wedge \exists_{4 \leq z}(\delta(z) \wedge \mathrm{C} z y \wedge \neg \mathrm{O} z y \wedge \forall u((\delta(u) \wedge u \neq y \wedge \neg \mathrm{O} u z) \rightarrow$ $\neg \mathrm{C} u z)) \wedge \exists u \exists z(\delta(u) \wedge \delta(z) \wedge \neg \mathrm{O} u z \wedge \mathrm{C} u z \wedge \mathrm{C} x u \wedge \mathrm{C} x z \wedge \mathrm{C} y u))$

Given any finite $L$-structure $\mathcal{A}$, we will extend it to a finite model $\mathcal{B}$ of SAGEMT via the following four steps.

(Step 1) For each $a \in \operatorname{Dom}(\mathcal{A})$, add ten new atoms $e_{1}$ to $e_{10}$ to $\operatorname{Dom}(\mathcal{B})$ and let $a$ be composed of $e_{1}$ and $e_{2}, a_{1}$ be composed of $e_{3}$ and $e_{4}, a_{2}$ be composed of $e_{5}$ and $e_{6}, a_{3}$ be composed of $e_{7}$ and $e_{8}$ and $a_{4}$ be composed of $e_{9}$ and $e_{10}$. Let $a$ contact $a_{1}, a_{2}, a_{3}$ and $a_{4}$, that is, let $\left(a, a_{1}\right),\left(a, a_{2}\right),\left(a, a_{3}\right)$ and $\left(a, a_{4}\right) \in C^{\mathcal{B}}$. The construction should be such that all the atoms newly added are all distinct and do not contact each other (this condition should also be met at Step 2 and Step 3).

(Step 2) For any $a \in \operatorname{Dom}(\mathcal{A})$, if $(a, a) \in R^{\mathcal{A}}$, that is, $R(a, a)$ is true in $\mathcal{A}$, then add two more new atoms $e_{11}$ and $e_{12}$ to $\operatorname{Dom}(\mathcal{B})$, let $a_{5}$ be composed of these two atoms and add $\left(a, a_{5}\right)$ to $C^{\mathcal{B}}$; otherwise, do nothing.

(Step 3) For any $a, b \in \operatorname{Dom}(\mathcal{A})$, if both $(a, b)$ and $(b, a) \in R^{\mathcal{A}}$, then add four new atoms $c_{1}, c_{2}, d_{1}$ and $d_{2}$ to $\operatorname{Dom}(\mathcal{B})$, and let $c$ be composed of $c_{1}$ and $c_{2}$ and $d$ be composed of $d_{1}$ and $d_{2}$ such that $c$ contacts $d$, and $a$ as well as $b$ contacts both $c$ and $d$, that is, add $(c, d),(a, c),(a, d)$, $(b, c)$ and $(b, d)$ to $C^{\mathcal{B}}$; if $(a, b) \in R^{\mathcal{A}}$ but $(b, a) \notin R^{\mathcal{A}}$, then add four new atoms $c_{1}, c_{2}, d_{1}$ and $d_{2}$ to $\operatorname{Dom}(\mathcal{B})$, and let $c$ be composed of $c_{1}$ and $c_{2}$ and $d$ be composed of $d_{1}$ and $d_{2}$ such that $c$ contacts $d, a$ contacts both $c$ and $d$, and $b$ contacts $c$ only, that is, add $(c, d),(a, c),(a, d)$ and $(b, c)$ to $C^{\mathcal{B}}$; otherwise, do nothing.

(Step 4) is exactly the same as Step 4 in the proof of the case of AGEMT.

Now let's check that the mereotopological structure $\mathcal{B}$ constructed via the foregoing four steps is a model of SAGEMT. First of all, for the same reason mentioned earlier, $\mathcal{B}$ satisfies AGEMT. Furthermore, from the definition of $C^{\mathcal{B}}$, it can be easily seen that for any $a, b$ in $\mathcal{B}$, if $a$ is an atom, then $(a, b) \in C^{\mathcal{B}}$ if and only if $(a, b) \in P^{\mathcal{B}}$. But since $\mathcal{B}$ is atomic, for any $a, b$ in $\mathcal{B}, a$ is not a part of $b$ if and only if $a$ has at least one atom which is not a part of $b$ (for a more rigorous argument, it is not difficult to show that $\mathbf{E M}+(\mathrm{A}) \models \mathrm{P} x y \leftrightarrow \forall z(($ At $z \wedge \mathrm{P} z x) \rightarrow \mathrm{P} z y)$, and $\mathcal{B}$ is 
of course a model of EM $+(A)$ ). From the said observations, it follows that for any $a, b$ in $\mathcal{B}, a$ is not a part of $b$ if and only if $a$ has at least one atom which does not contact $b$. By this biconditional, $\mathcal{B}$ obviously satisfies (C4). Hence $\mathcal{B}$ is a model of SAGEMT. Similar to the case of AGEMT, we can then conclude that SAGEMT is finitely inseparable and here let's skip the final details of how to reach that conclusion.

\section{Concluding remarks}

From the main result above, it follows that any mereotopological theory up to AGEMT or SAGEMT is hereditarily undecidable (since as mentioned earlier, any subtheory of a finitely inseparable theory must also be finitely inseparable) and strongly undecidable in the second sense (it can be seen immediately from the definitions involved that "finitely inseparable" implies "strongly undecidable in the second sense"). Moreover, any theory $T$ of such a kind must be separable since $T$ has at least one finite model and the theory of a finite model must be decidable, and for the same reason, $T$ is neither essentially undecidable nor strongly undecidable in the first sense (this is immediate from the definitions involved). How about those mereotopological theories each of which has no finite models? In fact, combining the results which I have already shown previously [16], we can come to the following two general conclusions.

1. All consistent mereotopological theories which can be generated from the axioms listed in the first section have decidable consistent extensions in the same language; therefore they are separable and are neither essentially undecidable nor strongly undecidable in the first sense.

2. All consistent mereotopological theories which can be generated from the axioms listed in the first section, except SAGEMTC or SAX ${ }^{\prime}$ or $\mathbf{S} \overline{\mathbf{B}} \mathbf{X}^{\prime}$, for any $\mathbf{X}$ strictly stronger than $\mathbf{C E M T}$, are hereditarily undecidable.

Finally, the following questions still remain open:

whether SAGEMTC is decidable or not; whether any of $\mathbf{S A X}^{\prime}$ or $\mathbf{S B} \mathbf{X}^{\prime}$, for any $\mathbf{X}$ strictly stronger than $\mathbf{C E M T}$, is decidable or not (I guess none of them is decidable).

Acknowledgments. This paper is a product kindly supported by National Science Council, Taiwan (NSC 101-2410-H-194-033-MY3). 


\section{References}

[1] Casati, R., and A. C. Varzi., Parts and Places, The MIT Press, 1999.

[2] Clarke, B. L., "A calculus of individuals based on 'connection' ", Notre Dame Journal of Formal Logic, 22 (1981): 204-218.

[3] Enderton, H. B., A Mathematical Introduction to Logic, San Diego, Academic Press, 2001.

[4] Hodges, W., Model Theory, Cambridge, Cambridge University Press, 1993.

[5] Monk, J. D., Mathematical Logic, New York, Springer-Verlag, 1976.

[6] Pratt-Hartmann, I., "First-order mereotopology", in Handbook of Spatial Logics, M. Aiello, I. Pratt-Hartmann and J. van Benthem (ed.), Dordrecht, Springer, 2007, pp. 13-97.

[7] Shoenfield, J. R., Mathematical Logic, London, Addison-Wesley, 1967.

[8] Simons, P., Parts: A Study in Ontology, Oxford, Clarendon Press, 1987.

[9] Soare, R., Recursively Enumerable Sets and Degrees, Perspectives in Mathematical Logic, Springer-Verlag, 1987.

[10] Tarski, A., A. Mostowski, and R. M. Robinson, Undecidable Theories, Amsterdam, North-Holland Publishing Company, 1953.

[11] Tarski, A., "On the foundations of Boolean algebra", in Logic, Semantics, Metamathematics, Oxford, Oxford University Press, 1956.

[12] Tsai, Hsing-chien, "Decidability of mereological theories", Logic and Logical Philosophy, 18 (2009): 45-63.

[13] Tsai, Hsing-chien, "More on the decidability of mereological theories", Logic and Logical Philosophy, 20 (2011): 251-265.

[14] Tsai, Hsing-chien, "Decidability of General Extensional Mereology", Studia Logica, 101 (2013), 3: 619-636. DOI: 10.1007/s11225-012-9400-4.

[15] Tsai, Hsing-chien, "A comprehensive picture of the decidability of mereological theories", Studia Logica, DOI: 10.1007/s11225-012-9405-z (2012).

[16] Tsai, Hsing-chien, "On the decidability of axiomatized mereotopological theories", Notre Dame Journal of Formal Logic (forthcoming 2013).

[17] Whitehead, A. N., Process and Reality, The MacMillan Company, New York, 1929.

HSING-CHIEN TSAI

Department of Philosophy

National Chung-Cheng University

168 University Road, Min-Hsiung

Chia-Yi, 62102 Taiwan

pythc@ccu.edu.tw 